\title{
ELASTIC PROBLEM OF A WIRE-WOUND CYLINDER
}

\author{
By Chester Snow
}

\section{ABSTRACT}

The elastic problem here treated is that of an infinitely long circular cylinder around whieh is wrapped an endless set of equal wires, equally spaced, with given tension. The deformation in cylinder and wires is found after determining the pressure distribution and the shape and size of the contact surface between a wire and the cylinder. The latter are found by a method which is a natural extension of Hertz's theory of contact. The contact surface is a ring of finite length instead of an infinitesimal ellipse as in Hertz's method so that the integral equation determining the pressure function and its range is in this case a logarithmic potential instead of a Newtonian one. Numerical application is made to measurements on a precision standard of inductance, which is a single layer coil on a porcelain cylinder. It is concluded that error's due to deformation of wire and cylinder will be negligible in the computation of the inductance provided that the over-all diameter is measured after winding.

\section{CONTENTS}

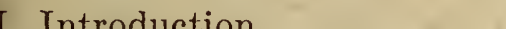

II. Fundamental equations

III. Formal solution for the wire $\ldots \ldots \ldots 336$

1. Particular solution for a single turn of wine $\ldots \ldots \ldots 36$

2. The stress function $\chi$ for the wire $\ldots$

IV. Formal solution for the cylinder $\ldots$

V. Determination of the pressure distribution and the contact surface $-\quad 350$

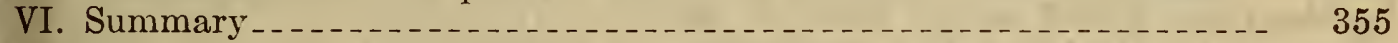

\section{INTRODUCTION}

A very long circular cylinder of known unstressed radius $a$ is uniformly wound with circular turns of wire of spacing $2 \pi p$. The unstressed radius of the wires $\rho_{\circ}$ and their tension $T$ being given, the deformation in wires and cylinder is desired. This problem arises in connection with absolute electrical measurements which are based upon the computed inductance of a single-layer solenoid. The solution here presented gives the elastic displacement and stresses to the first order inclusive in the small ratio $\frac{\rho_{\mathrm{o}}}{a}$. Formal solutions of the equations of elastic equilibrium in wires and cylinder being obtained, the satisfaction of the boundary conditions and the expression of contact lead to an integral equation (of the logarithmic potential type) for the determination of the pressure distribution between wire and cylinder and the shape and size of the contact dent. 


\section{FUNDAMENTAL EQUATIONS}

If $\sigma$ and $E$ denote Poisson's ratio and Young's modulus, respectively, the cylindrical components $u_{r} u_{\theta}$ and $u_{z}$ of the elastic displacement must satisfy the three equations of equilibrium

$$
\begin{aligned}
& \left.\frac{\partial \Delta}{\partial r}+(1-2 \sigma)\left\{\nabla^{2} u_{r}-\frac{u_{r}}{r^{2}}-\frac{2}{r^{2}} \frac{\partial u_{\theta}}{\partial \theta}\right\}=0\right\} \\
& \left.\frac{1}{r} \frac{\partial \Delta}{\partial \theta}+(1-2 \sigma)\left\{\nabla^{2} u_{\theta}-\frac{u_{\theta}}{r^{2}}+\frac{2}{r^{2}} \frac{\partial u_{r}}{\partial \theta}\right\}=0\right\} \\
& \frac{\partial \Delta}{\partial \mathrm{z}}+(1-2 \sigma) \Delta^{2} u_{z}=0
\end{aligned}
$$

where the dilatation $\Delta$ is

$$
\Delta=\frac{\partial u_{r}}{\partial r}+\frac{u_{r}}{r}+\frac{1}{r} \frac{\partial u \theta}{\partial \theta}+\frac{\partial u_{z}}{\partial z}
$$

The stress components are derived from the displacement by the formulas

$$
\begin{aligned}
& \overparen{r r}=\frac{E}{1+\sigma}\left\{\frac{\sigma}{1-2 \sigma} \Delta+\frac{\partial u_{r}}{\partial r}\right\} \ldots . . r_{z}=\frac{E}{2(1+\sigma)}\left\{\frac{\partial u_{z}}{\partial r}+\frac{\partial u_{r}}{\partial z}\right\} \\
& \overparen{z z}=\frac{E}{1+\sigma}\left\{\frac{\sigma}{1-2 \sigma} \Delta+\frac{\partial u_{z}}{\partial z}\right\} \ldots . \widehat{r \theta}=\frac{E}{2(1+\sigma)}\left\{\frac{\partial u_{\theta}}{\partial r}-\frac{u_{\theta}}{r}+\frac{1}{r} \frac{\partial u_{r}}{\partial \theta}\right\} \\
& \overparen{\theta \theta}=\frac{E}{1+\sigma}\left\{\frac{\sigma}{1-2 \sigma} \Delta+\frac{u_{r}}{r}+\frac{1}{r} \frac{\partial u_{\theta}}{\partial \theta}\right\}--\overparen{z \theta}=\frac{E}{2(1+\sigma)}\left\{\frac{\partial u_{\theta}}{\partial z}+\frac{1}{r} \frac{\partial u_{z}}{\partial \theta}\right\}
\end{aligned}
$$

In the case where $u_{\theta}=0$ while $u_{r}$ and $u_{z}$ are independent of $\theta$, a displacement satisfying the equations of equilibrium may be derived from a stress function $\chi$ which satisfies

$$
\nabla^{4} x \equiv \nabla^{2} \nabla^{2} \chi=0 \text { (where } \chi \text { is independent of } \theta \text { ) }
$$

by the formulas

$$
\left.\begin{array}{c}
\frac{E}{1+\sigma} u_{r}=-\frac{\partial^{2} \chi}{\partial r \partial z} \\
\frac{E}{1+\sigma} u_{z}=2(1-\sigma) \nabla^{2} \chi-\frac{\partial^{2} \chi}{\partial z^{2}}
\end{array}\right\}
$$

In this case the dilatation is given by

$$
\frac{E}{1+\sigma} \cdot \frac{\sigma}{1-2 \sigma} \Delta=\sigma \frac{\partial}{\partial z} \nabla^{2} \chi
$$


and the stress components are

$$
\left.\begin{array}{l}
\widehat{r r}=\frac{\partial}{\partial z}\left[\sigma \nabla^{2} \chi-\frac{\partial^{2} \chi}{\partial r^{2}}\right] \ldots \widehat{r z}=\frac{\partial}{\partial r}\left[(1-\sigma) \nabla^{2} \chi-\frac{\partial^{2} \chi}{\partial z^{2}}\right] \\
\widehat{z z}=\frac{\partial}{\partial z}\left[(2-\sigma) \nabla^{2} \chi-\frac{\partial^{2} \chi}{\partial z^{2}}\right] \ldots \widehat{r \theta}=0 \\
\widehat{\theta \theta}=\frac{\partial}{\partial z}\left[\sigma \nabla^{2} \chi-\frac{1}{r} \frac{\partial \chi}{\partial r}\right] \ldots \widehat{\partial \theta}=0
\end{array}\right\}
$$

The foregoing equations are appropriate in considering the stress and strain in the cylinder.

In considering the elastic equilibrium of a torus or single turn of circular wire which is wrapped tightly around an elastic circular cylinder whose unstressed radius is $a$, we have to find a displacement satisfying the equations of equilibrium and such that the stresses derived from it reduce to a normal pressure at the boundary of the wire. It is convenient to use in this case the coordinates $\rho, \beta$, and $\theta$ instead of the cylindrical coordinates $r, z$, and $\theta$ where $\rho, \beta$ are plane polar coordinates of any section of the wire made by a plane through the $z$-axis. The single turn of wire when unstressed is the torus gererated by rotating about the $z$-axis the circle whose equation in the $r, z$ plane (or any plane $\theta$-const) is $\left(r-a_{0}\right)^{2}+z^{2}=\rho_{0}^{2}$ where $\rho_{0}$ is the radius of the wire and $a_{0}$ is the initial $r$-coordinate of the center of its section and less than $a+\rho_{0}$. The origin for the plane polar coordinates is the point $r=a_{0}, z=0$, so that the equation of the wire section when unstressed is $\rho=\rho_{0}$. The angle $\beta$ will be taken as zero at the point of the section nearest the $z$-axis and will range from $-\pi$ to $\pi$. (See fig. 1.) We may imagine the very long elastic circular cylinder of unstressed radius $a$ which is greater than $a_{0}-\rho_{0}$, subjected to a uniform hydrostatic pressure on its convex surface, which is sufficient to compress its radius from $a$ to $a_{0}-\rho_{0}$ so that it will just slip into the ring of wire. When this is done and the two ends of the cylinder are a great distance from the wire, the hydrostatic pressure may be imagined to be removed from the cylinder, which, by expansion, puts the wire under tension whose average value $\bar{T}=\frac{1}{\pi \rho_{0}^{2}} \iint T d S$ taken over the wire section will be considered as known and this will ultimately determine $a_{0}$.

Any particle of the wire whose coordinates were $\rho, \beta, \theta$, moves to a point whose coordinates are $\rho+u_{\rho}(\rho, \beta), \beta+\frac{1}{\rho} u_{\beta}(\rho, \beta), \theta+\frac{1}{r} u_{\theta}(\rho, \beta, \theta)$, where the plane polar coordinates $\rho, \beta$, are referred to the original center of the wire section as origin, which is the point $r=a_{0}, z=0$. The passage from the coordinates $\rho, \beta$, to the cylindrical coordinates $r, z$ is made by

$$
r=a_{0}-\rho \cos \beta \text { and } z=\rho \sin \beta
$$

and the displacement is transformed by the formulas

$$
\left.\begin{array}{l}
u_{\rho}=-u_{r} \cos \beta+u_{z} \sin \beta \\
u_{\beta}=u_{r} \sin \beta+u_{z} \cos \beta
\end{array}\right\} \text { or }\left\{\begin{array}{l}
u_{r}=-u_{\rho} \cos \beta+u_{\beta} \sin \beta \\
u_{z}=u_{z} \sin \beta+u_{\beta} \cos \beta
\end{array}\right.
$$


The boundary conditions to be satisfied are that at the surface of the wire where $\rho=\rho_{0}$, the stresses become

$$
\begin{aligned}
& \widehat{\rho \rho}=-P(\beta)=-1 / 2 P_{0}-\sum_{n=1}^{\infty} P_{n} \cos n \beta \\
& \widehat{\rho \beta}=0 \\
& \widehat{\rho \theta}=0
\end{aligned}
$$

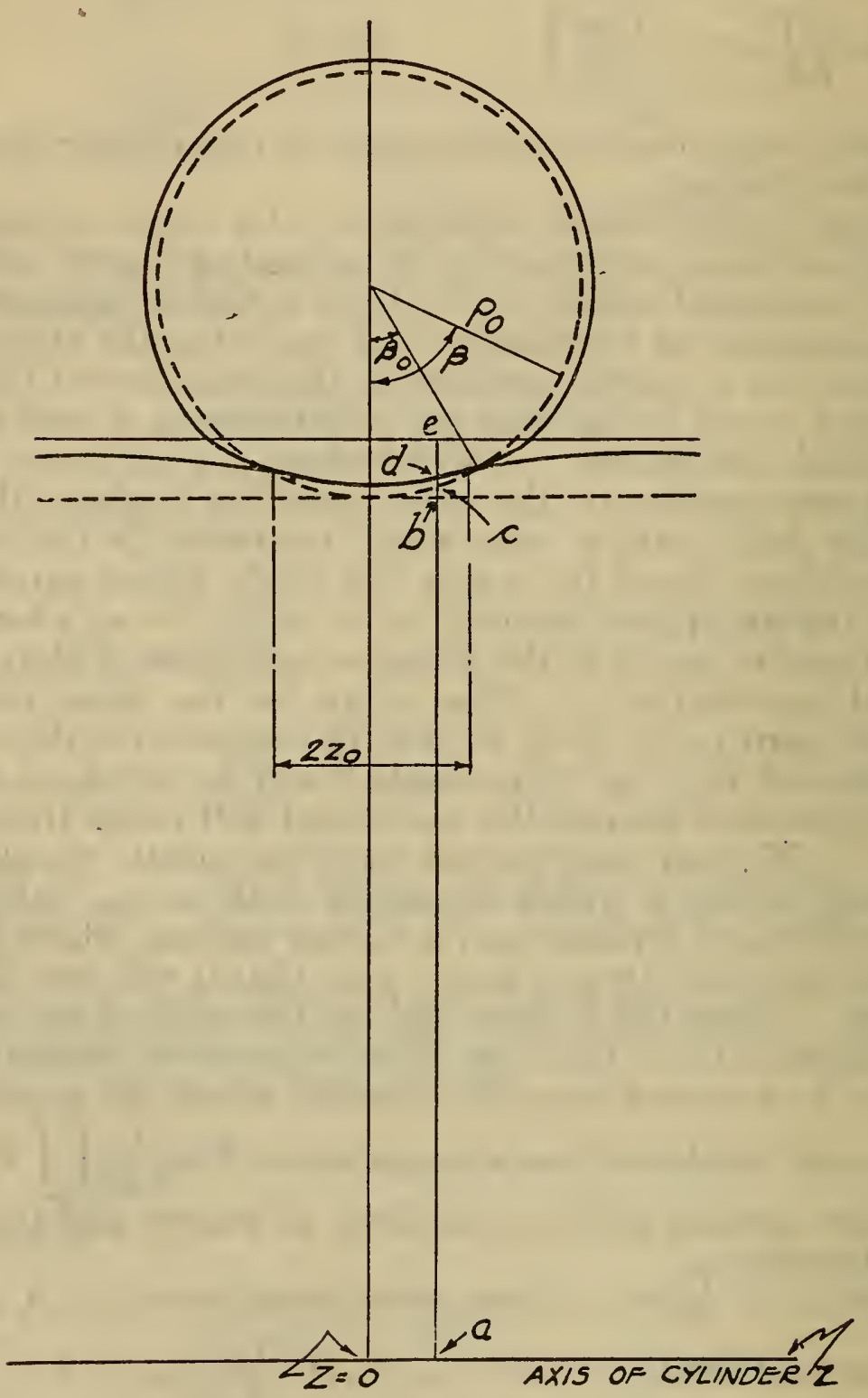

FIGURE 1.-Section of one wire and cylinder by a plane through the cylindrical axis

Heavy lines $=$ section when stressed. Dotted circle $=$ section of unstressed wire. Dotted straight line $=$ section of cylinder when uniformly compressed. $\overline{a b}=a_{0}-\rho_{0}=$ radius of cylinder when compressed to just slip in the ring of wire. $\overline{a e}=a=$ radius of cylinder before compression. $\overline{a d}=r$ $(z)=$ radius of the contact surface at point $z$.

where $P(\beta)$ is the unknown normal pressure exerted by the cylinder upon the wire. It is evidently an even function of and may be written

$$
P(\beta)=1 / 2 P_{0}+\sum_{n=1}^{\infty} P_{n} \cos n \beta_{\ldots} \ldots \ldots-\pi \leqq \beta \leqq \pi
$$


where

$$
P_{n}=\frac{1}{\pi} \int_{\pi}^{\pi} P(\beta) \cos n \beta d \beta=\frac{1}{\pi} \int_{\beta_{0}}^{\beta_{0}} P(\beta) \cos n \beta d \beta
$$

The last integral expresses the fact that the contact ring has the range $-\beta_{0}<\beta<\beta_{0}$, since outside this range of $\beta$ there is nothing touching the surface of the wire. This angle $\beta_{0}$ is also unknown and must be determined by the solution of the problem of elastic equilibrium of the two bodies in contact. Since $\beta_{0}$ is obviously a small fraction of $\pi$, it is evident that the coefficients $P_{n}$ will not differ appreciably for small values of $n$. These preliminaries are in no way altered if we have an infinite number of equally spaced wires wrapped around an infinitely long cylinder. The stress and strain in each wire will be a duplicate of that in the one we shall consider whose center remains in the plane $z=0$.

The stress components are derived from the displacement by the general formulas

$$
\begin{gathered}
\widehat{\rho \rho}=\frac{E}{1+\sigma}\left[\frac{\sigma \Delta}{1-2 \sigma}+\frac{\partial u_{\rho}}{\partial \rho}\right] \ldots \widehat{\rho \beta}=\frac{E}{2(1+\sigma)}\left[\rho \frac{\partial}{\partial \rho}\left(\frac{u_{\beta}}{\rho}\right)+\frac{\partial}{\partial \beta}\left(\frac{u_{\rho}}{\rho}\right)\right] \\
\beta \beta=\frac{E}{1+\sigma}\left[\frac{\sigma \Delta}{1-2 \sigma}+\frac{1}{\rho}\left(u_{\rho}+\frac{\partial u_{\beta}}{\partial \beta}\right]\right. \\
\widehat{\rho \theta}=\frac{E}{2(1+\sigma)}\left[r \frac{\partial}{\partial \rho}\left(\frac{u_{\theta}}{r}\right)+\frac{\partial}{\partial \theta}\left(\frac{u_{\rho}}{r}\right)\right] \ldots \theta=\frac{E}{1+\sigma}\left[\frac{\sigma \Delta}{1-2 \sigma}\right. \\
\left.+\frac{1}{r}\left(u_{r}+\frac{u_{\theta}}{\partial \theta}\right)\right] \ldots \beta \theta=\frac{E}{2(1+\sigma)}\left[\frac{1}{r} \frac{\partial u_{\beta}}{\partial \theta}-\frac{u_{\theta} \sin \beta}{r}+\frac{1}{\rho} \frac{\partial u_{\theta}}{\partial \beta}\right]
\end{gathered}
$$

where the dilatation is

$$
\Delta=\frac{\partial u_{\rho}}{\partial \rho}+\frac{u \rho}{\rho}+\frac{1}{\rho} \frac{\partial u_{\beta}}{\partial \beta}+\frac{u_{\tau}}{r}+\frac{1}{r} \frac{\partial u_{\theta}}{\partial \theta}
$$

According to equation (9), the formulas for transforming directional derivatives are

$$
\left.\begin{array}{l}
\frac{\partial}{\partial \rho}=-\cos \beta \frac{\partial}{\partial r}+\sin \beta \frac{\partial}{\partial z} \\
\frac{1}{\rho} \frac{\partial}{\partial \beta}=\sin \beta \frac{\partial}{\partial r}+\cos \beta \frac{\partial}{\partial z}
\end{array}\right\} \text { or }\left\{\begin{array}{l}
\frac{\partial}{\partial r}=-\cos \beta \frac{\partial}{\partial \rho}+\frac{\sin \beta}{\rho} \frac{\partial}{\partial \beta} \\
\frac{\partial}{\partial z}=\sin \beta \frac{\partial}{\partial \rho}+\frac{\cos \beta}{\rho} \frac{\partial}{\partial \beta}
\end{array}\right.
$$

When $u_{\theta}=0$ and $u_{r}$ and $u_{z}$ are functions of $r$ and $z$ only (that is, of $\rho$ and $\beta$ ), then the stress function $\chi$ is a function of $\rho$ and $\beta$, and the $\rho$ and $\beta$ components of the displacement are found by use of (15) in the equations (5). These give

$$
\begin{aligned}
& \frac{E}{1+\sigma} u_{\rho}=2(1-\sigma) \sin \beta \nabla^{2} \chi-\frac{\partial}{\partial \rho}\left(\frac{\partial \chi}{\partial z}\right) \\
& \frac{E}{1+\sigma} u_{\beta}=2(1-\sigma) \cos \beta \nabla^{2} \chi-\frac{1}{\rho} \frac{\partial}{\partial \beta}\left(\frac{\partial \chi}{\partial z}\right)
\end{aligned}
$$


where $\frac{\partial \chi}{\partial z}$ is merely the abbreviation for $\sin \beta \frac{\partial \chi}{\partial \rho}+\frac{\cos \beta}{\rho} \frac{\partial \chi}{\partial \beta}$ as in (15). Similarly we find in this way from (13) the following formulas for the stress components

$$
\begin{aligned}
& \rho \theta=\beta \theta \equiv 0 \\
& \rho \rho=\sigma \frac{\partial}{\partial z} \Delta^{2} \chi+\frac{E}{1+\sigma} \frac{\partial u_{\rho}}{\partial \rho} \\
& \rho \beta=(1-\sigma)\left[\cos \beta \frac{\partial}{\partial \rho}+\frac{\sin \beta}{\rho} \frac{\partial}{\partial \beta}\right] \nabla^{2} \chi-\frac{\partial}{\partial \rho}\left[\frac{1}{\rho} \frac{\partial}{\partial \beta} \cdot \frac{\partial \chi}{\partial z}\right] \\
& \theta \theta=\sigma \frac{\partial}{\partial z} \nabla^{2} \chi+\frac{E}{1+\sigma} \frac{u_{r}}{r} \\
& \beta \beta=\sigma \frac{\partial}{\partial z} \nabla^{2} \chi+\frac{E}{1+\sigma} \cdot \frac{1}{\rho}\left(u_{\rho}+\frac{\partial u_{\beta}}{\partial \beta}\right)
\end{aligned}
$$

We have in mind applications to the case where the ratio $\frac{\rho_{0}}{a}$ is small (0.002) and shall have to consider displacements which are the sum of terms of the three relative orders of magnitude $\frac{a^{2}}{\rho_{0}^{2}}, \frac{a}{\rho_{0}}$, and 1 , although all three will be small due to a common factor $E^{-1}$ which is of the order of $10^{-12}$.

\section{FORMAL SOLUTION FOR THE WIRE}

In this section a formal solution will be constructed for points in a wire, which satisfy the elastic equations and boundary conditions, but which contain in their expression certain undetermined constants and the unknown pressure function and its range. In the next section the same is done for points in the cylinder, and in Section $V$ the two solutions are brought together in the statement of contact, by which all the unknown constants and pressure distribution will be determined.

As a matter of convenience, a particular solution will be presented and then a more general type of solution which is derivable from a stress function $\chi$. The particular solution is not a special case of the latter, but their sum gives a solution with a sufficient number of constants to satisfy the boundary conditions.

\section{PARTICULAR SOLUTION FOR A SINGLE TURN OF WIRE}

It is found that whatever the constants $A \ldots \ldots A_{4}$, the following displacement represents a particular solution of the equations of elastic equilibrium (1).

$$
\begin{aligned}
& \frac{E}{1+\sigma} u_{\theta}^{\prime}=A r \theta \\
& \frac{E}{1+\sigma} u_{r}^{\prime}=\left(A+A_{1}\right) r+\frac{a_{0}^{2} A_{2}+A_{4} z^{2}}{r} \\
& \frac{E}{1+\sigma} u_{2}^{\prime}=2(1-2 \sigma)\left(A-A_{4}\right) z \log r+A_{3} z
\end{aligned}
$$


If we take

$$
\begin{aligned}
\epsilon & \equiv \frac{\rho_{0}}{4 a_{0}} \\
A_{1} & =\frac{1-5 \sigma-2 \sigma^{2}}{2 \sigma(1+\sigma)} A \\
A_{2} & =-A_{4}=\frac{1-2 \sigma}{2 \sigma} A \\
A_{3} & =\left[\frac{1-\sigma}{1+\sigma}+\frac{1-2 \sigma}{\sigma} \log a_{0}\right] A \\
A & =\frac{\sigma}{1-\sigma} \cdot \frac{C}{4 \epsilon}
\end{aligned}
$$

then expanding to the second order in $\epsilon$ we find the stresses $\widehat{\rho \theta}^{\prime} \equiv 0$

$$
\begin{gathered}
\widehat{\rho \rho^{\prime}}=-\epsilon C \frac{9-14 \sigma}{4(1-\sigma)}\left(\frac{\rho}{\rho_{0}}\right)^{2}-C \frac{\rho}{\rho_{0}} \cos \beta-\epsilon C \frac{1-2 \sigma}{2(1-\sigma)}\left(\frac{\rho}{\rho_{0}}\right)^{2} \cos 2 \beta \\
-\epsilon C \frac{3(1-2 \sigma)}{4(1-\sigma)}\left(\frac{\rho}{\rho_{0}}\right)^{2} \cos 4 \beta \\
\widehat{\rho \beta^{\prime}}=\epsilon C \frac{1-2 \sigma}{2(1-\sigma)}\left(\frac{\rho}{\rho_{0}}\right)^{2} \sin 2 \beta+\epsilon C \frac{3(1-2 \sigma)}{4(1-\sigma)}\left(\frac{\rho}{\rho_{0}}\right)^{2} \sin 4 \beta \\
\widehat{\theta \theta}=\frac{C}{4 \epsilon}+\epsilon C \frac{2-5 \sigma}{1-\sigma}\left(\frac{\rho}{\rho_{0}}\right)^{2}+C \frac{1-3 \sigma}{1-\sigma} \frac{\rho}{\rho_{0}} \cos \beta+\epsilon C \frac{4-9 \sigma}{1-\sigma}\left(\frac{\rho}{\rho_{0}}\right)^{2} \cos 2 \beta
\end{gathered}
$$

The displacement is then given by

$$
\begin{aligned}
& \frac{E}{1+\sigma} \frac{u_{\theta^{\prime}}}{a_{0}}=\theta \frac{\sigma}{1-\sigma}\left[\frac{C}{4 \epsilon}-C \frac{\rho}{\rho_{0}} \cos \beta\right] \\
& \frac{E}{1+\sigma} \frac{u_{r}^{\prime}}{a_{0}}=\frac{1-2 \sigma-\sigma^{2}}{1-\sigma^{2}} \cdot \frac{C}{4 \epsilon}+\frac{\sigma C}{1+\sigma} \frac{\rho}{\rho_{0}} \cos \beta+2 \epsilon C \frac{1-2 \sigma}{1-\sigma}\left(\frac{\rho}{\rho_{0}}\right)^{2} \cos 2 \beta \\
& \frac{E}{1+\sigma} \frac{u_{2}^{\prime}}{a_{0}}=-\frac{\sigma C}{1+\sigma} \frac{\rho}{\rho_{0}} \sin \beta-2 \epsilon C \frac{1-2 \sigma}{1-\sigma}\left(\frac{\rho}{\rho_{0}}\right)^{2} \sin 2 \beta
\end{aligned}
$$

The $\rho$ and $\beta$ components of $u^{\prime}$ are given by

$$
\left.\begin{array}{rl}
\frac{E}{1+\sigma} \frac{u_{\rho}^{\prime}}{a_{0}} & =-\frac{\sigma C}{1+\sigma} \cdot \frac{\rho}{\rho_{0}}-\left[\frac{1-2 \sigma-\sigma^{2}}{1-\sigma^{2}} \cdot \frac{C}{4 \epsilon}+2 \epsilon C \frac{1-2 \sigma}{1-\sigma}\left(\frac{\rho}{\rho_{0}}\right)^{2}\right] \cos \beta \\
\frac{E}{1+\sigma} \frac{u_{\beta}}{a_{0}} & =\left[\frac{1-2 \sigma-\sigma^{2}}{1-\sigma^{2}} \cdot \frac{C}{4 \epsilon}-2 \epsilon C \frac{1-2 \sigma}{1-\sigma}\left(\frac{\rho}{\rho_{0}}\right)^{2}\right] \sin \beta
\end{array}\right\}
$$

The solution is being considered for the single turn of wire whose center remains in the plane $z=0$. The stress and strain in all other wires are repetitions of this. 


\section{THE STRESS FUNCTION $\chi$ FOR THE WIRE}

Using $\rho, \beta$ instead of the cylindrical coordinates $r, z$, it is readily found that a solution of the equation (4) to the first order inclusive in $\epsilon$ is

$$
\chi=\rho_{0}{ }^{3} \sum_{n=1}^{\infty}\left\{D_{n}\left(\frac{\rho}{\rho_{0}}\right)^{n}+B_{n}\left(\frac{\rho}{\rho_{0}}\right)^{n+2}+\epsilon B_{n \mathrm{H}}\left(\frac{\rho}{\rho_{0}}\right)^{n+4}\right\} \sin n \beta
$$

To avoid duplicating certain terms included in the particular solution, we shall here take $D_{1}=D_{2}=0$. The constants $D_{n}$ and $B_{n}$ are assumed to be of the same order of magnitude as the pressure applied to the wire. The corresponding value of $\nabla^{2} \chi$ is to the first order.

$$
\begin{aligned}
\nabla^{2} \chi= & 4 \rho_{0} \sum_{n=1}^{\infty}\left\{\left(\frac{\rho}{\rho_{0}}\right)^{n}\left[(n+1)\left(B_{n}-\epsilon D_{n+1}\right)-\epsilon B_{n-1}\right]\right. \\
& \left.+(n+2) \epsilon B_{n+1}\left(\frac{\rho}{\rho_{0}}\right)^{n+2}\right\} \sin n \beta
\end{aligned}
$$

The displacement is then found by use of formulas (16)

$$
\begin{aligned}
& \frac{\mathrm{E}}{1+\sigma} \cdot \frac{u_{\rho}}{\rho_{0}}=-\epsilon\left\{6 B_{1}\left(\frac{\rho}{\rho_{0}}\right)^{2}+4(1-\sigma)\left[D_{1}-2 B_{1}\left(\frac{\rho}{\rho_{0}}\right)^{2}\right]\right\} \cos \beta \\
&-\sum_{n=0}^{\infty} \cos n \beta\left\{( \frac { \rho } { \rho _ { 0 } } ) ^ { n - 1 } \left[n(n+1) D_{n+1}+(3-4 \sigma) n B_{n-1}\right.\right. \\
&\left.-4 \epsilon(1-\sigma)\left(n D_{n}+B_{n-2}\right)\right]+\left(\frac{\rho}{\rho_{0}}\right)^{n+1}(n+2)[(n-2 \\
&\left.+4 \sigma) B_{n+1}+2 \epsilon\left((1-2 \sigma) B_{n}+2(1-\sigma) D_{n+2}\right)\right] \\
&\left.+\left(\frac{\rho}{\rho_{0}}\right)^{n+3}(n+3)(n+4 \sigma) \epsilon B_{n+2}\right\} \\
& \frac{E}{1+\sigma} \frac{u_{\beta}}{\rho_{0}}= \epsilon\left\{2 B_{1}\left(\frac{\rho}{\rho_{0}}\right)^{2}+4(1-\sigma)\left[D_{1}-2 B_{1}\left(\frac{\rho}{\rho_{0}}\right)^{2}\right]\right\} \sin \beta \\
&+ \sum_{n=1}^{\infty} \sin n \beta\left\{( \frac { \rho } { \rho _ { 0 } } ) ^ { n - 1 } \left[n(n+1) D_{n+1}+(3-4 \sigma) n B_{n-1}\right.\right. \\
&\left.-4(1-\sigma) \epsilon\left(n D_{n}+B_{n-2}\right)\right]+\left(\frac{\rho}{\rho_{0}}\right)^{n+1}\left[(n+2)(n+4-4 \sigma) B_{n+1}\right. \\
&+\left.2 \epsilon\left((1-2 \sigma)_{n} B_{n}-2(1-\sigma)(n+2) D_{n+2}\right)\right]+\left(\frac{\rho}{\rho_{0}}\right)^{n+3} \\
&\left.(n+3)(n+4-4 \sigma) \epsilon B_{n+2}\right\}
\end{aligned}
$$


(where it is understood that $D_{n} \equiv 0$ if $n<3$ and $\mathrm{B}_{n}=0$ if $n<1$ ). These expressions enable one by formulas (17) to compute the stresses. It is found that

$$
\begin{aligned}
\widehat{\rho \beta}= & {\left[6 B_{2} \frac{\rho}{\rho_{0}}+16(2-\sigma) \epsilon B_{3}\left(\frac{\rho}{\rho_{0}}\right)^{3}\right] \sin \beta+\sum_{n=2}^{\infty} \sin n \beta\{(n-1)[n(n+1)} \\
& \left.D_{n+1}+(3-4 \sigma) n B_{n-1}-4(1-\sigma) \epsilon\left(n D_{n}+B_{n-2}\right)\right]\left(\frac{\rho}{\rho_{0}}\right)^{n-2} \\
+ & n(n+1)\left[(n+2) B_{n+1}+2(1-2 \sigma) \epsilon B_{n}\right]\left(\frac{\rho}{\rho_{0}}\right)^{n}+(n+3) \\
& {\left.[(n+1)(n+2)+2(1-2 \sigma)] \epsilon B_{n+2}\left(\frac{\rho}{\rho_{0}}\right)^{n+2}\right\} }
\end{aligned}
$$

and

$$
\begin{aligned}
\widehat{\rho \rho} & =4 B_{1}-12 \sigma \epsilon B_{2}\left(\frac{\rho}{\rho_{0}}\right)^{2}+\left\{\left[6 B_{2}-24 \epsilon D_{3}-8 \epsilon B_{1}\right] \frac{\rho}{\rho_{0}}\right. \\
& \left.-16(1+\sigma) \epsilon B_{3}\left(\frac{\rho}{\rho_{0}}\right)^{3}\right\} \cos \beta-\sum_{n=2}^{\infty} \cos n \beta\left\{( n - 1 ) \left[n(n+1) D_{n+1}\right.\right. \\
& \left.+(3-4 \sigma) n B_{n-1}-4(1-\sigma) \epsilon\left(n D_{n}+B_{n-2}\right)\right]\left(\frac{\rho}{\rho_{0}}\right)^{n-2} \\
& +(n+1)\left[(n+2)(n+2) B_{n+1}+\epsilon\left(4(n+2) D_{n+2}+2(2+(1\right.\right. \\
& \left.\left.-2 \sigma) n) B_{n}\right)\right]\left(\frac{\rho}{\rho_{0}}\right)+(n+3)[(n+1)(n+2) \\
& \left.-2(1-2 \sigma)] \epsilon B_{n+2}\left(\frac{\rho}{\rho_{0}}\right)^{n+2}\right\}
\end{aligned}
$$

$$
\text { (Also } \widehat{\rho \theta} \equiv 0)
$$

These give the stress components to the first order inclusive in $\epsilon$.

The boundary conditions (10) may be satisfied by the sum of the particular solution of the preceding section and the solution here obtained. We, therefore, add to the $\widehat{\rho \beta}$ component given by (29) the component $\widehat{\rho}^{\prime}$ given by $(21)$, and after placing $\rho=\rho_{0}$, equate the sum to zero. This gives

$$
B_{2}=-\frac{8}{3}(2-\sigma) \epsilon B_{3}
$$

and for $n>2$ (remembering that $D_{1} \equiv D_{2} \equiv 0$ )

$$
\begin{aligned}
D_{n}= & -(3-4 \sigma) \frac{B_{n-2}}{n}-\frac{n+1}{n-2} B_{n}+\epsilon\left\{4(1-\sigma)\left(\frac{D_{n-1}}{n}+\frac{B_{n-3}}{(n-1) n}\right)\right. \\
& -\frac{2(1-2 \sigma)}{n-2} B_{n-1}-\frac{(n+2)[n(n+1)+2(1-2 \sigma)] B_{n+1}}{(n-2)(n-1) n} \\
& \left.-\frac{1-2 \sigma}{4(1-\sigma)} C t_{n}\right\}
\end{aligned}
$$


where

$$
\begin{aligned}
t_{n} & =\frac{1}{3} \text { if } n=3 \\
& =\frac{1}{20} \text { if } n=5 \\
& =0 \text { otherwise }
\end{aligned}
$$

The remaining boundary condition is satisfied by adding to the expression (30) for $\rho \rho$ the corresponding expression (20) for $\rho \rho^{\prime}$ and equating their sum when $\rho=\rho_{0}$ to $-P(\beta)$. Doing this and making use of the corresponding equations $(31)^{1}$ and $(31)^{n}$ gives, on equating coefficients of $\cos n \beta$,

and for $n>2$

$$
\begin{aligned}
& 4 B_{1}-4 \epsilon\left[3 \sigma B_{2}+\frac{9-14 \sigma}{16(1-\sigma)} C\right]=-\frac{1}{2} P_{0} \\
& -C-8 \epsilon\left[\mathrm{B}_{1}+3 D_{3}+6 B_{3}\right]=-P_{1}
\end{aligned}
$$

$$
B_{n}=-\frac{1}{2} \frac{P_{n-1}}{n(n+1)}+2 \epsilon\left[D_{n+1}+\frac{B_{n-1}}{n+1}-\frac{(1-2 \sigma)(n+2) B_{n+1}}{n(n+1)}\right]
$$

Since the two sets of equation (31) and (32) are valid to the first order in $\epsilon$ inclusive, we solve them by placing

$$
\left.\begin{array}{l}
B_{n}=B_{n}{ }^{0}+\epsilon B_{n}{ }^{\prime} \text { for } n=1,2,3, \cdots \\
D_{n}=D_{n}{ }^{0}+\epsilon D_{n}{ }^{\prime} \text { for } n=3,4,5, \cdots \\
C=C^{0}+\epsilon C^{\prime}+\epsilon^{2} C^{\prime \prime}
\end{array}\right\}
$$

where $B_{n}{ }^{0}, B_{n}{ }^{\prime}$, etc., are assumed to be of the same order as the $\mathrm{P}_{n}$. Each equation of the two sets then gives two relations, by equating finite parts and by equating first-order parts. The constant $C^{\prime \prime}$ will appear only in $(32)^{1}$ and can not be determined by these equations, since the left side of $(32)^{1}$ neglects second-order terms in $\epsilon$ so that the retention of $\epsilon^{2} C^{\prime \prime}$ there would not be justifiable.

We find by equating finite terms in equation $(31)^{1},(32)^{0}$, and $(32)^{\mathbf{n}}$ that

$$
\begin{aligned}
& B_{1}{ }^{0}=-\frac{1}{8} P_{0} \\
& B_{2}{ }^{0}=0 \\
& B_{n}{ }^{0}=-\frac{1}{2} \frac{P_{n-1}}{n(n+1)} \text { if } n>2
\end{aligned}
$$

The finite terms in $(32)^{1}$ give

$$
C^{0}=P_{1}
$$


Using these values the finite parts of $(31)^{n}$ then give (since $D_{1} \equiv D_{2} \equiv 0$ )

$$
\left.\begin{array}{l}
D_{3}{ }^{0}=\frac{3-4 \sigma}{2} \frac{1}{1 \cdot 2 \cdot 3} \cdot \frac{P_{0}}{2}+\frac{1}{2}\left(\frac{P_{2}}{1 \cdot 3}\right) \\
D_{4}{ }^{0}= \\
D_{n}{ }^{0}=\frac{3-4 \sigma}{2}\left(\frac{P_{3}}{2 \cdot 4}\right) \\
\frac{P_{n-3}}{(n-2)(n-1) n}+\frac{1}{2} \frac{P_{n-1}}{(n-2) n} \text { if } n>4
\end{array}\right\}
$$

Using these values and next equating first order terms in $(32)^{\circ}$ and $(32)^{1}$ gives

$$
\left.\begin{array}{rl}
\epsilon C^{\prime} & =-2 \epsilon\left[(1-2 \sigma) P_{0}+P_{2}\right] \\
\epsilon B_{1}^{\prime} & =\frac{9-14 \sigma}{16(1-\sigma)} \epsilon P_{1}
\end{array}\right\}
$$

The remaining constants $\epsilon D_{n}^{\prime}$ and $\epsilon B^{\prime}$ may now be found by equating first-order terms in all the equations of the two sets, but their evaluation would be useless unless $C$ were known with even greater precision than is represented by the retention of the terms $\epsilon^{2} C^{\prime \prime}$. In fact, if this term $\epsilon^{2} C^{\prime \prime}$ could not be found, the values of $B_{\mathrm{n}}^{\circ}$ and $\dot{D}_{\mathrm{n}}^{\circ}$ here obtained would be useless for it, like them, contributes first-order terms to the displacement, but, unlike them, it contributes only second-order terms to the $\widehat{\rho \rho}$ - and $\widehat{\rho \beta}$-components of stress. We consider, however, that the average tension $\bar{T}$ in the wire is given. It is defined by

$$
\bar{T}=\frac{1}{\pi \rho_{0}^{2}} \int_{0}^{\rho_{0}} \rho d \rho \int_{-\pi}^{\pi} \widehat{\theta \theta} d \beta
$$

To compute $\bar{T}$ we note that the $\widehat{\theta \theta}$-component of stress, which is derivable from the stress function $\chi$ is

$$
\sigma \frac{\partial}{\partial z} \nabla^{2} \chi+\frac{E}{1+\sigma} \frac{u_{r}}{r}
$$

so that by the expressions (26), (27), and (28) we find that $\chi$ contributes to the tension the amount

$$
\bar{T} \chi=8 \sigma B_{1}+12(1+\sigma) \epsilon B_{2}=-\sigma P_{0}+\frac{\sigma(18-28 \sigma)}{4(1-\sigma)} \epsilon P_{1}
$$

Also from (22) the particular solution contributes

$$
\overline{T^{\prime}}=\frac{C}{4 \epsilon}+\frac{\epsilon C(4-10 \sigma)}{4(1-\sigma)}=\frac{P_{1}}{4 \epsilon}-\frac{P_{0}+P_{2}}{2}+\sigma P_{0}+\frac{\epsilon}{4}\left[C^{\prime \prime}+\frac{4-10 \sigma}{1-\sigma} P_{1}\right]
$$

Hence we find

$$
\begin{gathered}
\bar{T}=\frac{P_{1}}{4 \epsilon}-\frac{P_{0}+P_{2}}{2}+\frac{\epsilon}{4}\left[C^{\prime \prime}+\frac{4\left(1+2 \sigma-7 \sigma^{2}\right)}{1-\sigma} P_{1}\right] \\
=\frac{C}{4 \epsilon}-\sigma P_{0}+\frac{1+2 \sigma-7 \sigma^{2}}{1-\sigma} \epsilon P_{1}
\end{gathered}
$$

$64825-31-9$ 
Since $\bar{T}$ is absolutely given, this equation determines $C^{\prime \prime}$ in terms of $\bar{T}, P_{0}, P_{1}$, and $P_{2}$. This term $C^{\prime \prime}$ only enters the expression for the displacement in the factor $\frac{C}{4 \epsilon}$ so it is more convenient to avoid the explicit use of $\epsilon^{2} C^{\prime \prime}$ and use the formula

$$
\frac{C}{4 \epsilon}=\bar{T}+\sigma P_{0}-\frac{1+2 \sigma-7 \sigma^{2}}{1-\sigma} \epsilon P_{1}
$$

If we now use the values given above of $B_{\mathrm{n}}^{0}$ and $D_{\mathrm{n}}^{0}$, we find for that part of the displacement in the wire which is derivable from the stress-function $\chi$; that is, the finite terms of (27) and (28).

$$
\begin{gathered}
u_{\rho}(\rho, \beta)=-\frac{\rho_{0}(1+\sigma)}{2 E}\left\{(1-2 \sigma) \frac{\rho}{\rho_{0}} P_{0}+\sum_{n=2}^{\infty} P_{n}\left[\frac{n}{n-1}\left(\frac{\rho}{\rho_{0}}\right)^{\mathrm{n}-1}\right.\right. \\
\left.\left.-\frac{n+1-3+4 \sigma}{n+1}\left(\frac{\rho}{\rho_{0}}\right)^{\mathrm{n}+1}\right] \cos n \beta\right\} \\
u_{\beta}(\rho, \beta)=\frac{\rho_{0}(1+\sigma)}{2 E} \sum_{n=2}^{\infty} P_{n}\left[\frac{n}{n-1}\left(\frac{\rho}{\rho_{0}}\right)^{n-1}\right. \\
\left.-\frac{n+1+3-4 \sigma}{n+1}\left(\frac{\rho}{\rho_{0}}\right)^{n+1}\right] \sin n \beta
\end{gathered}
$$

If we introduce the integral (12) for the Fourier coefficient $P_{n}$ and sum the series, these expressions are transformed into integrals, and we find for that part of the displacement in the wire derivable from $\chi$

$$
\begin{aligned}
& u_{\rho}(\rho, \beta)=\frac{1+\sigma}{E}\left\{(1-\sigma) \rho P_{0}+\left[\frac{3-4 \sigma}{4}\left(\frac{\rho}{\rho_{0}}\right)^{2}\right.\right. \\
&\left.-2(1-\sigma) \log \rho_{0}\right] \rho_{0} P_{1} \cos \beta \\
&-\frac{\rho}{\rho_{0}}\left(1-\frac{\rho^{2}}{\rho_{2}}\right) \frac{\rho_{0}^{2}}{2 \pi} \int_{-\beta_{0}}^{\beta_{0}}\left(\beta^{1}\right)\left(1-\frac{2 \rho_{0}}{\rho} \sin \psi\right) \frac{\cos \psi}{R} d \beta^{\prime} \\
&-\frac{(1-2 \sigma)}{\pi} \rho_{0} \int_{-\beta_{0}}^{\beta_{0}}\left(\beta^{\prime}\right) \psi \sin \left(\beta-\beta^{\prime}\right) d \beta^{\prime} \\
&\left.+\frac{2(1-\sigma) \rho_{0}}{\pi} \int_{-\beta_{0}}^{\beta_{0}}\left(\beta^{\prime}\right) \cos \left(\beta-\beta^{\prime}\right) \log R d \beta^{\prime}\right\} \\
& u_{\beta}(\rho, \beta)= \frac{1+\sigma}{E}\left\{\left[\frac{3-4 \sigma}{4}\left(\frac{\rho}{\rho_{0}}\right)^{2}+2(1-\sigma) \log \rho_{0}\right] \rho_{0} P_{1} \sin \beta\right. \\
&-\frac{\rho}{\rho_{0}}\left(1-\frac{\rho^{2}}{\rho_{0}^{2}}\right) \frac{\rho_{0}^{2}}{2 \pi} \int_{-\beta_{0}}^{\beta_{0}} P \beta^{\prime}\left(1-2 \frac{\rho}{\rho_{0}} \cos \left(\beta-\beta^{\prime}\right)\right) \frac{\sin \psi}{R} d \beta^{\prime} \\
&-\frac{(1-2 \sigma) \rho_{0}}{\pi} \int_{-\beta_{0}}^{\beta_{0}} P\left(\beta^{\prime}\right) \psi \cos \left(\beta-\beta^{\prime}\right) d \beta^{\prime} \\
&\left.-\frac{2(1-\sigma) \rho_{0}}{\pi} \int_{-\beta_{0}}^{\beta_{0}} P\left(\beta^{\prime}\right) \sin \left(\beta-\beta^{\prime}\right) \log R d \beta^{\prime}\right\}
\end{aligned}
$$


where $R$ is the distance from the point of integration $\rho_{0}, \beta^{\prime}$ upon the arc of the section of wire, to the point $\rho, \beta$ within, and $\psi$ is the angle between $R$ and the interior normal, so that

$$
R^{2}=\rho^{2}-2 \rho \rho_{0} \cos \left(\beta-\beta^{\prime}\right)+\rho_{0}^{2} \text { and }\left\{\begin{array}{l}
\mathrm{R} \cos \psi=\rho_{0}-\rho \cos \left(\beta-\beta^{\prime}\right) \\
\mathrm{R} \sin \psi=\rho \sin \left(\beta-\beta^{\prime}\right)
\end{array}\right.
$$

When the point $\rho, \beta$ approaches as a limit any point $\rho_{0}, \beta$ on the circular boundary of the wire section, then .

$$
\begin{aligned}
R & =2 \rho_{0}\left|\sin \frac{\beta-\beta^{\prime}}{2}\right| \\
\psi & =-\frac{1}{2}\left(\beta-\beta^{\prime}\right)+\frac{\pi}{2} \text { if } \beta-\beta^{\prime}>0 \\
& =-\frac{1}{2}\left(\beta-\beta^{\prime}\right)-\frac{\pi}{2} \text { if } \beta-\beta^{\prime}<0
\end{aligned}
$$

Resolving the displacement in the direction of increasing $r$, we find from (43) and (44) the following expression for the $u_{r}$ which is due to the stress function

$$
\begin{gathered}
u_{r}(\rho, \beta) \equiv-\frac{1+\sigma}{E}\left\{\frac{2(1-\rho) P_{0}}{\pi} \int_{-\beta_{0}}^{\circ} P\left(\beta^{\prime}\right) \cos \beta^{\prime} \log R d \beta^{\prime}\right. \\
+\frac{(1-2 \sigma) p_{0}}{\pi} \int_{-\beta_{0}}^{\beta_{0}} P\left(\beta^{\prime}\right) \sin \beta^{\prime} \psi d \beta^{\prime} \\
+\frac{\rho}{\rho_{0}}\left(1-\frac{\rho^{2}}{\rho_{0}^{2}}\right) \frac{\rho_{0}^{2}}{2 \pi} \int_{\beta_{0}-}^{\beta_{0}} \frac{P\left(\beta^{\prime}\right)}{R}\left[\cos (\beta+\psi)+2 \frac{\rho}{\rho_{0}} \sin \psi\left(\sin \left(\beta-\beta^{\prime}\right) \sin \beta\right.\right. \\
-\cos \beta \text { us } \psi)] d \beta^{\prime}-2(1-\sigma) \log \rho_{0} \cdot \rho_{0} P_{1}+(1-\sigma) \rho P_{0} \cos \beta+\frac{3-4 \sigma}{4}\left(\frac{\rho}{\rho_{0}}\right)^{2} \\
\left.\rho_{0} P_{1} \cos \beta\right\}
\end{gathered}
$$

When the point $\rho, \beta$, is on the contact ring, $\beta^{2}$ like $\beta^{\prime 2}$ becomes negligible and (49) reduces to

$$
\begin{gathered}
u_{r}\left(\rho_{0}, \beta\right)=-\frac{1+\sigma}{E}\left\{\frac{2(1-\sigma) \rho_{0}}{\pi} \int_{-\beta_{0}}^{\beta_{0}} P_{\left(\beta^{\prime}\right)} \log R d \beta^{\prime}+(1-\sigma) \rho_{0} P_{0}\right. \\
\left.+\left[\frac{3-4 \sigma}{4}+2(1-\sigma) \log \rho_{0}\right] \rho_{0} P_{1}\right\}
\end{gathered}
$$

Since this represents only the contribution of the function $\chi$, we must add to it the corresponding component given by equation (23) for the particular solution. Using the value of $C$ we have found, this is

$$
\begin{aligned}
& u_{r}\left(\rho_{0}, \beta\right)=\frac{a_{0}}{E(1-\sigma)}\left\{\left(1-2 \sigma-\sigma^{2}\right)\left(\bar{T}+\sigma P_{0}\right)+\sigma(1-\sigma) P_{1}\right\} \\
& +\frac{\rho_{0}}{4 E}\left\{-2 \sigma\left[(1-2 \sigma) P_{0}+P_{2}\right]\right. \\
& \left.+\frac{2\left(1-\sigma^{2}\right)(1-2 \sigma)-\left(1-2 \sigma-\sigma^{2}\right)\left(1+2 \sigma-7 \sigma^{2}\right)}{(1-\sigma)^{2}} P_{1}\right\}
\end{aligned}
$$


The total $u_{r}$ component on the contact surface is the sum of (50) and (51).

Since $\rho_{0}$ is a very small angle, we may write $z_{0}=\rho_{0} \beta_{0}$ and $z=\rho_{0} \beta$ so that the definitions (12) for $P_{n}$ may be written

$$
P_{n}=\frac{1}{\pi} \int_{-\beta_{0}}^{\beta_{0}} P\left(\beta^{\prime}\right) \cos n \beta^{\prime} d \beta^{\prime}=\frac{1}{\pi \rho_{0}} \int_{-z_{0}}^{z_{0}} P\left(z^{\prime}\right) \cos \frac{n z^{\prime}}{\rho_{0}} d z^{\prime}
$$

Since the width $2 z_{0}$ of the contact strip is obviously very small compared with the radius $\rho_{0}$ of the wire, we may here write $\cos \frac{n z^{\prime}}{\rho_{0}}=1$ $-\frac{1}{2}\left(\frac{n z^{\prime}}{\rho_{0}}\right)^{2}$ and for small values of $n$, such as $n=1$ or 2 , this is equal to 1 to the first order, inclusive. That is, the approximations already made do not justify us in distinguishing between $P_{0}, P_{1}$, and $P_{2}$, and require that we place

$$
P_{0}=P_{1}=P_{2}
$$

If we denote by $\lambda_{1}, \lambda_{2}$, and $\lambda_{3}$ the three positive elastic constants which depend only upon the dimensionless $\sigma$

$$
\begin{aligned}
& \lambda_{1} \equiv \frac{1-2 \sigma-\sigma^{2}}{(1+\sigma)(1-\sigma)^{2}}, \lambda_{2} \equiv \frac{\sigma\left(2-3 \sigma-\sigma^{2}\right)}{(1+\sigma)(1-\sigma)^{2}} \\
& \lambda_{3} \equiv \frac{6-7 \sigma-21 \sigma^{2}+35 \sigma^{3}-5 \sigma^{4}}{4(1+\sigma)(1-\sigma)^{3}}
\end{aligned}
$$

we then find by addition of (50) and (51) for the $r$ component of the total displacement of those particles of the wire which are on the contact surface, where $\rho=\rho_{0}$ and $-z_{0}<z=\rho_{0} \beta<z_{0}$

where

$$
\begin{gathered}
u_{\tau}=c\left\{a_{0}\left[\lambda_{1} \bar{T}+\lambda_{2} P_{0}\right]-\rho_{0} P_{0}\left[\lambda_{3}-2 \log \rho_{0}\right]\right. \\
\left.-\frac{2}{\pi} \int_{-z_{0}}^{z_{0}} P\left(z^{\prime}\right) \log \left|z-z^{\prime}\right| d z^{\prime}\right\}
\end{gathered}
$$

$c \equiv \frac{1-\sigma^{2}}{E}$

when

$$
\sigma=1 / 3, \lambda,=3 / 8, \lambda_{z}=1 / 2 \text { and } \lambda_{3}=15 / 8
$$

so that these three positive constants are of ordinary magnitude. All of the terms in the second member of (54) are small, due to the factor $c$ which is of the order of $10^{-12}$ absolute cgs. units. Since $a_{0}$ is very nearly $a-\rho_{0}$ it is evident that the terms in $\rho_{0} P_{0}$ and the integral are small compared to the first term, and the tension $\bar{T}$ contributes the greater part of this term.

It is important to recall what is our hypothesis as to the initial or unstressed shape of the wire in order to properly interpret the dis- 
placement (54). The initial $r$ coordinate of any point $z$ on the surface of the wire which later comes into contact with the cylinder was

$$
a_{0}-\rho_{0}+\rho_{0}(1-\cos \beta)=a_{0}-\rho_{0}+\frac{\rho_{0} \beta^{2}}{2}=a_{0}-\rho_{0}+\frac{z^{2}}{2 \rho_{0}}
$$

Therefore, when equilibrium is attained, its $r$ coordinate is

$$
r(z)=a_{0}-\rho_{0}+\frac{z^{2}}{2 \rho_{0}}+u_{r}
$$

Or using (54)

$$
\begin{gathered}
r(z)=a_{0}-\rho_{0}+\frac{z^{2}}{2 \rho_{0}}+c\left\{a_{0}\left[\lambda_{1} \bar{T}+\lambda_{2} P_{0}\right]-\rho_{0} P_{0}\left[\lambda_{3}-2 \log \rho_{\theta}\right]\right. \\
\left.-\frac{2}{\pi} \int_{-Z_{0}}^{Z_{0}}\left(z^{\prime}\right) \log \left|z-z^{\prime}\right| d z^{\prime}\right\}
\end{gathered}
$$

The small quantity $\left(\frac{z_{0}}{\rho_{0}}\right)^{2}$ like $\left(\frac{z}{\rho_{0}}\right)^{2}$ is by no means negligible here, but its neglect in those terms of (57) which contain the factor $c$ was justified since $c$ is of the order of $10^{-12}$. The unknowns in the second member of (57) are $a_{0}, P_{0}, z_{0}$ and the pressure distribution $P(z)$. When the second member of this equation is known, it becomes the equation of the curve which is a section of the surface of contact, by a plane through the $z$ axis. We may obtain another independent integral relation between $\bar{T}, r(z)$ and $P(z)$ by considering the equilibrium of a segment of the wire included between two planes through the $z$ axis corresponding to $\theta=\theta_{1}$ and $\theta=-\theta_{1}$. At each circular section the total tension $T$ acts on the part of the wire considered and the two amount to a force $2 T \sin \theta_{1}=2 \pi \rho_{0}{ }^{2} T \sin \theta_{1}$, directed toward the $z$ axis. This force is counter balanced by the component in the opposite direction of the pressure exerted by the cylinder upon the segment of the wire which is

$$
\int_{-\theta_{1}}^{\theta_{1}} \cos \theta d \theta \int_{-z_{0}}^{z_{0}} P(z) r(z) d z=2 \sin \theta_{1} \int_{-z_{0}}^{z_{0}} P(z) r(z) d z
$$

whence

$$
2 T \sin \theta_{1}=2 \sin \theta_{1} \pi \rho_{0}^{2} \bar{T}=2 \sin \theta_{0} \int_{-z_{0}}^{z_{0}}(z) r(z) d z
$$

so that

$$
\frac{1}{\pi \rho_{0}^{2}} \int_{-z_{0}}^{z_{0}} r(z) P(z) d z=\bar{T}
$$

This relation will be used after we know more about the form of the function $r(z)$. 


\section{FORMAL SOLUTION FOR THE CYLINDER}

In this section a formal solution of the elastic equations and boundary conditions will be constructed for points in the cylinder. Like that obtained above for the wire, its expression will involve the unknown pressure distribution and its range.

In this case the boundary conditions to be satisfied by the stresses at $r=a$ are

$$
\begin{aligned}
& \widehat{r z}=0 \\
& \widehat{r \theta}=0 \\
& \widehat{r r}=-P(z)=-\left\{\frac{1}{2} f_{0}+\sum_{n=1}^{\infty} f_{\mathrm{n}} \cos \frac{n z}{p}\right\} \text { for }-\infty<z<+\infty
\end{aligned}
$$

where

$$
f_{\mathrm{n}}=\frac{1}{\pi p} \int_{-\pi p}^{\pi p} \underset{-\pi}{\pi p}(z) \cos \frac{n z}{p} d z=\frac{1}{\pi p} \int_{-z_{0}}^{z_{0}}(z) \cos \frac{n z}{p} d z
$$

Since $2 \pi p$ is the distance between centers of the endless set of wires, the pressure $P(z)$ must be a periodic function of $z$ with period $2 \pi p$. The Fourier's Series in (59) for $P(z)$ has a different range from the corresponding development (11) and hence the coefficients $P_{n}$ and $f_{n}$ are different. For the coefficients $P_{n}$ defined by (12) may be written (letting $\beta=\frac{z}{\rho_{0}}$ )

$$
P_{n}=\frac{1}{n \rho_{0}} \int_{-z_{0}}^{z_{0}} \stackrel{\cos }{\rho_{\rho_{0}}}(z) \cos z
$$

which shows by comparison with (60) in the particular case, $n=0$, the relation

$$
p f_{0}=\rho_{0} P_{0}=\frac{1}{\pi} \int_{-z_{0}}^{z_{0}}(z) d z
$$

We shall be able to satisfy the boundary condition (59) by a displacement which at all points in the cylinder is derivable from a stress function $x$ satisfying the equation (4). A stress function which is a particularly simple solution of (4)

$$
\chi_{0}=\frac{f_{0}}{4(1+\sigma)}\left[(1-\sigma) r^{2} z-\frac{2}{3}(2-\sigma) z^{3}\right]
$$

gives rise to the displacement by (7)

$$
\begin{aligned}
& u_{\tau}^{0}=-\frac{1-\sigma}{2 E} f_{0} r \\
& u_{\theta}^{0}=0 \\
& u_{2}^{0}=\frac{\sigma}{E} f_{0} z
\end{aligned}
$$


with the stress components

$$
\widehat{r r}=\widehat{\theta \theta}=-\frac{1}{2} f_{0}=\text { constant }
$$

All other stress components are identically zero, and, in particular, the cylinder is not under longitudinal tension, $\overparen{z z} \equiv 0$. If $J_{0}$ and $J_{1}$ denote Bessel's function, we begin with a solution of equation (4) of the form

$$
\chi=\chi_{0}+\sum_{n=1}^{\infty}\left\{A_{n} J_{0}\left(\frac{i n r}{p}\right)+B_{n} \frac{i n r}{p} J_{1}\left(\frac{i n r}{p}\right)\right\} \sin \frac{n z}{p}
$$

which gives

$$
\nabla^{2} \chi=-\frac{f_{0} z}{1+\sigma}-2 \sum_{n=1}^{\infty} \frac{n^{2}}{p^{2}} B_{n} J_{0}\left(\frac{i n r}{p}\right) \sin \frac{n z}{p}
$$

This gives by the formula (6) the dilatation

$$
\frac{E}{1+\sigma} \cdot \frac{\sigma}{1-2 \sigma} \Delta=\sigma \frac{\partial}{\partial z} \nabla^{2} x=-\sigma\left\{\frac{f_{0}}{1+\sigma}+2 \sum_{n=1}^{\infty} \frac{n^{3}}{p^{3}} B_{n} J_{0}\left(\frac{i n r}{p}\right) \cos \frac{n z}{p}\right\}
$$

Hence, the formulas (7) give the stress components

$$
\begin{aligned}
\widehat{r \theta}=0 & \\
\widehat{r z} & =i \sum_{n=1}^{\infty} \frac{n^{3}}{p^{3}} \sin \frac{n z}{p}\left\{A_{n} J_{1}\left(\frac{i n r}{p}\right)-B_{n}\left[\frac{i n r}{p} J_{0}\left(\frac{i n r}{p}\right)+2(1-\sigma) J_{1}\left(\frac{i n r}{p}\right)\right]\right\} \\
\widehat{r r} & =-\frac{1}{2} f_{0}-\sum_{n=1}^{\infty} \frac{n^{3}}{p^{3}} \cos \frac{n z}{p}\left\{A_{n}\left[J_{0}\left(\frac{i n r}{p}\right)-\frac{J_{1}\left(\frac{i n r}{p}\right)}{i n r / p}\right]\right. \\
& \left.\quad-B_{n}\left[(1-2 \sigma) J_{0}\left(\frac{i n r}{p}\right)-\frac{i n r}{p} J_{1}\left(\frac{i n r}{p}\right)\right]\right\}
\end{aligned}
$$

If we let

$$
\alpha=\frac{n a}{p}
$$

the vanishing of $\widehat{r} z$ at the boundary is assured by taking

$$
A_{n}=\frac{i \alpha J_{0}(i \alpha)+2(1-\sigma) J_{1}(i \alpha)}{J_{1}(i \alpha)} B_{n}
$$

Using this relation and satisfying the remaining boundary condition of (59) we find

$$
\begin{aligned}
& A_{n}=a \frac{p^{2}}{n^{2}} \frac{2(1-\sigma) i J_{1}(i \alpha)-\alpha J_{0}(i \alpha)}{\left(J_{0}^{2}(i \alpha)+J_{1}^{2}(i \alpha)\right)-2(1-\sigma) J_{1}^{2}(i \alpha)} \cdot f_{n} \\
& B_{n}=a \frac{p^{2}}{n^{2}} \frac{i J_{1}(i \alpha)}{(i \alpha)^{2}\left(J_{0}^{2}(i \alpha)+J_{1}^{2}(i \alpha)\right)-2(1-\sigma) J_{1}{ }^{2}(i \alpha)} f_{n}
\end{aligned}
$$


All the stress and strain components in the cylinder may now be found. In particular we find by (5) for the boundary value of $u_{r}$

$u_{r}(a, z)=-a c^{\prime}\left\{\frac{f_{\mathrm{o}}}{2\left(1+\sigma^{\prime}\right)}+2 \sum_{n=1}^{\infty} \frac{f_{\mathrm{n}} \cos \frac{n z}{\mathrm{p}}}{(i a)^{2}\left[\left(\frac{J_{0}(i a)}{J_{1}(i a)}\right)^{2}+1\right]-2\left(1-\sigma^{\prime}\right)}\right\}$

where

$$
c^{\prime} \equiv \frac{1-\sigma^{2}}{E^{\prime}} \text { and } \sigma^{\prime}, E^{\prime}
$$

are now used for $\sigma, E$ to distinguish between the constants of the wire and the cylinder.

When $\frac{p}{a}$ is less than 5 , reference to tables of Bessel's functions show that there will be an errór of less than 1 part in 10,000 made by using the asymptotic expression for these functions, which enables us to write since $a=\frac{n a}{p}$

$$
(i a)^{2}\left[\left(\frac{J_{0}(i a)}{J_{1}(i a)}\right)^{2}+1\right]=\frac{n a}{p}+1
$$

neglecting terms of order of $\frac{p}{a}$ which is 0.001 when $2 \pi p=0.1$ and $a=15$. Hence, we replace the exact expression (72) by

$$
u_{r}(a, z)=-a c^{\prime}\left\{\frac{f_{0}}{2\left(1+\sigma^{\prime}\right)}+\frac{2 p}{a} \sum_{n=1}^{\infty} \frac{f_{n} \cos \frac{n z}{p}}{n-\left(1-2 \sigma^{\prime}\right) \frac{p}{a}}\right\}
$$

or since we neglect $\frac{p^{2}}{a^{2}}$ in comparison with 1

$$
u_{\tau}(a, z)=-a c^{\prime}\left\{\frac{f_{0}}{2\left(1+\sigma^{\prime}\right)}+\frac{2 p}{a} \sum_{n=1}^{\infty} \frac{f_{n} \cos \frac{n z}{p}}{n}\right\}
$$

If we now place

$$
f_{0}=\frac{\rho_{0} P_{0}}{p} \text { by }(61) \text { and } f_{n}=\frac{1}{\pi p} \int_{z_{0}}^{z_{0}} P\left(z^{\prime}\right) \cos \frac{n z^{\prime}}{p} d z^{\prime}
$$

this becomes

$$
\begin{array}{r}
u_{r}(a, z)=-c^{\prime}\left\{\frac{a \rho_{0} P_{0}}{2 p\left(1+\sigma^{\prime}\right)}+\frac{1}{\pi} \int_{-z}^{z_{0}} P\left(z^{\prime}\right) d z^{\prime} \sum_{n=1}^{\infty} \frac{2 \cos \frac{n z}{p} \cos \frac{n z^{\prime}}{p}}{n}\right\} \\
=-c^{\prime}\left\{\frac{a \rho_{0} P_{0}}{2 p\left(1+\sigma^{\prime}\right)}+\frac{1}{\pi} \int_{-z_{0}}^{z_{0}} P\left(z^{\prime}\right) d z^{\prime} \sum_{n=1}^{\infty} \frac{\cos \frac{n}{p}\left(z+z^{\prime}\right)+\cos \frac{n}{p}\left(z-z^{\prime}\right)}{n}\right\} \\
=-c^{\prime}\left\{\frac{a \rho_{0} P_{0}}{2 p\left(1+\sigma^{\prime}\right)}+\frac{2}{\pi} \int_{-z_{0}}^{z z_{0}^{\prime \prime 2}} P\left(z^{\prime}\right) d z^{\prime} \sum_{n=1}^{\infty} \frac{1}{n} \cos \frac{n}{p}\left(z-z^{\prime}\right)\right\}
\end{array}
$$


since $P\left(z^{\prime}\right)$ is an even function of $z^{\prime}$. Now if $-x$-denotes the absolute value of $x$

$$
\sum_{n=1}^{\infty} \frac{1}{n} \cos \frac{n}{p}\left(z-z^{\prime}\right)=-\log \left|2 \sin \frac{z-z^{\prime}}{2 p}\right|
$$

Hence (76) becomes

$$
\begin{aligned}
u_{r}(a, z)=-c^{\prime}\left\{\left[\frac{a}{2 p\left(1+\sigma^{\prime}\right)}+\right.\right. & +2 \log p] \rho_{0} P_{0} \\
& \left.-\frac{2}{\pi} \int_{-z_{0}}^{z_{0}} P\left(z^{\prime}\right) \log \left|2 p \sin \frac{z-z^{\prime}}{2 p}\right| d z^{\prime}\right\}
\end{aligned}
$$

The negative of the second member gives the shrinkage in radius of the cylinder at any point $z$. The constant term represents a uniform shrinkage of radius, the variable part is a periodic function of $z$ which is small compared to the constant term.

When the point $(a, z)$ is inside the contact ring both $\frac{z}{p}$ and $\frac{z^{\prime}}{p}$ are of order of magnitude of $\frac{z_{0}}{p}$ and their squares are negligible so that (77) becomes for $-z_{0}<z<z_{0}$

$$
\begin{aligned}
u_{r}(a, z)=-c^{\prime}\left\{\left[\frac{a}{2 p\left(1+\sigma^{\prime}\right)}+2 \log p\right] \rho_{0} P_{0}\right. \\
\left.\quad-\frac{2}{\pi} \int_{-z_{0}}^{z_{0}} P\left(z^{\prime}\right) \log \left|z-z^{\prime}\right| d z^{\prime}\right\}
\end{aligned}
$$

Since the particle $z$ to which this refers, had the coordinate $r=a$ when the cylinder was unstressed, the distance of this particle from the $z$ axis when equilibrium is attained is $r(z)=a+u_{\tau}(a, z)$. Using (77) this gives for the deformed shape to the cylinder the equation

$$
\begin{aligned}
r(z)=a-c^{\prime}\left\{\left[\frac{a}{2 p\left(1+\sigma^{\prime}\right)}+\right.\right. & 2 \log p] \rho_{0} P_{0} \\
& \left.-\frac{2}{\pi} \int_{-z_{0}}^{z_{0}} P\left(z^{\prime}\right) \log \left|2 p \sin \frac{z-z^{\prime}}{2 p}\right| d z^{\prime}\right\}
\end{aligned}
$$

which holds for all values of $z$ and is a periodic function of $z$ with period $2 \pi p$ which is the distance between centers of the endless series of wires, all alike and uniformly spaced, which are wrapped tightly around the cylinder, whose unstressed radius was $a$. The $z$ coordinates of the wire centers are $z=0 \pm 2 \pi p k$ where $k$ is an integer.

When the point $z$ on the surface of the cylinder is on the contact area, of say that wire whose center is at $z=0$ the deformation of the cylinder is then given by

$$
\begin{aligned}
r(z)=a-c^{\prime}\left\{\left[\frac{a}{2 p\left(1+\sigma^{\prime}\right)}+2 \log p\right]\right. & \rho_{0} P_{0} \\
& \left.-\frac{2}{\pi} \int_{-z_{0}}^{z_{0}} P\left(z^{\prime}\right) \log \left|z-z^{\prime}\right| d z^{\prime}\right\}
\end{aligned}
$$




\section{DETERMINATION OF THE PRESSURE DISTRIBUTION AND THE CONTACT RANGE}

The Sections III and IV have been concerned with the construction of formal solutions giving the stress and strain in the wires and cylinder which satisfy the boundary conditions, but which contain in their expression the unknown pressure distribution $P(z)$, the range of contact $2 z_{0}$, the constant $P_{0}$, and the constant $a_{0}$, the latter being the distance of the center of a circular turn of wire from the $z$ axis before the (compressed) cylinder was inserted. In this section we take over these results, and since the ideas here involved are different we number the equations anew in order to concentrate upon the present argument.

The equation of the contact surface, or dent in the cylinder, Section IV, equation (80), we rewrite as

$r(z)=a-c^{\prime}\left[\frac{a}{2 p\left(1+\sigma^{\prime}\right)}+2 \log p\right] \rho_{0} P_{0}+\frac{2 c^{\prime}}{\pi} \int_{-z_{0}}^{z_{0}} P\left(z^{\prime}\right) \log \left|z-z^{\prime}\right| d z^{\prime}$

The equation of the dent in the wire, Section III, equation (57) is

$$
\begin{aligned}
r(z)=a_{0}-\rho_{0} & +\frac{z^{2}}{2 \rho_{0}}+c\left[a_{0}\left(\lambda_{1} \bar{T}+\lambda_{2} P_{0}\right)-\left(\lambda_{3}-2 \log \rho_{0}\right) \rho_{0} P_{0}\right] \\
& -\frac{2 c}{\pi} \int_{-z_{0}}^{z_{0}} P\left(z^{\prime}\right) \log \left|z-z^{\prime}\right| d z^{\prime}
\end{aligned}
$$

where by Section IV, equation (61)

$$
\rho_{0} P_{0}=\frac{1}{\pi} \int_{-z_{0}}^{z_{0}} P\left(z^{\prime}\right) d z^{\prime}
$$

and by Section III, equation (58)

$$
T=\pi \rho_{0}^{2} \bar{T}=\int_{-z_{0}}^{z_{0}} r(z) P(z) d z
$$

The fact of contact is implied by $r(z)$ being the same in equations (1) and (2). Eliminating it gives the integral equation to determine the pressure distribution $P(z)$ and its contact range $2 z_{0}$

$$
-2 \int_{-z_{0}}^{z_{0}} \frac{2\left(c+c^{\prime}\right) \rho_{0}}{z_{0}} P\left(z^{\prime}\right) \log \left|z-z^{\prime}\right| d z^{\prime}=A-\pi \frac{z^{2}}{z_{0}}
$$

where the constant $A$ (independent of $z$ ) is

$$
\begin{gathered}
A=\frac{2 \pi \rho_{0}}{z_{0}}\left\{a+\rho_{0}-a_{0}\left(1+\lambda_{1} c \bar{T}\right)-\left[c\left(\frac{a_{0} \lambda_{2}}{\rho_{0}}-\lambda_{3}+2 \log \rho_{0}\right)\right.\right. \\
\left.\left.+c^{\prime}\left(\frac{a}{2 p\left(1+\sigma^{\prime}\right)}+2 \log p\right)\right] \rho_{0} P_{0}\right\}
\end{gathered}
$$


To solve (5) we recall that the logarithmic potential at any point $z$ on a thin cylindrical strip of width $2 z_{0}$ upon which there is a surface density of electricity of amount $\sqrt{1-\left(\frac{z}{z_{0}}\right)^{2}}$ is

$$
-2 \int_{-z_{0}}^{z_{0}} \sqrt{1-\left(\frac{z^{\prime}}{z_{0}}\right)^{2}} \log \left|z-z^{\prime}\right| d z^{\prime}=\pi z_{0}\left(\frac{1}{2}+\log \frac{2}{z_{0}}\right)-\frac{\pi z^{2}}{z_{0}}
$$

Comparing this with (5) shows that the solution of that equation is obtained by imposing the two conditions

$$
P(z)=\frac{\sqrt{z_{0}^{2}-z^{2}}}{2\left(c+c^{\prime}\right) \rho_{0}} \text { for }-z_{0}<z<z_{0}
$$

and

$$
A=\pi z_{0}\left(\frac{1}{2}+\log \frac{2}{z_{0}}\right)
$$

If $A$ were known (9) would determine $z_{0}$ and (8) then give $P(z)$. However, $A$ is a function of the unknown $z_{0}, a_{0}$, and $P_{0}$ as shown by (6) so that (6) and (9), together by elimination of $A$ give one relation between these three unknown constants from which $a_{0}$ may be later found. The equations (1) and (2) will thus be rendered compatible by (6), (8), and (9). The next equation (3) becomes by the use of (8)

$$
\rho_{0} P_{0}=\frac{1}{2 \pi \rho_{0}\left(c+c^{\prime}\right)} \int_{-z_{0}}^{z_{0}} \sqrt{z_{0}^{2}-z^{2}} d z=\frac{z_{0}^{2}}{4 \rho_{0}\left(c+c^{\prime}\right)}
$$

which may be written

$$
\frac{z_{0}}{\rho_{0}}=\sqrt{4\left(c+c^{\prime}\right) P_{0}}
$$

To satisfy the remaining condition (4) we first replace the integral in (1) by its value from (5). This gives by use of (10)

$$
r(z)=a\left(1-b c^{\prime} P_{0}\right)+\frac{c^{\prime} z^{2}}{2\left(c+c^{\prime}\right) \rho_{0}}=a\left\{1-c^{\prime} P_{0}\left[b-\frac{2 \rho_{0}}{a}\left(\frac{z}{z_{0}}\right)^{2}\right]\right\}
$$

where

$$
b=\frac{\rho_{0}}{2 p\left(1+\sigma^{\prime}\right)}+\frac{\rho_{0}}{a}\left(1+\log \frac{\rho^{2}}{\left(c+c^{\prime}\right) \rho_{0}^{2} P_{0}}\right)
$$

The equation (4), therefore, becomes

$$
\pi \rho_{0}^{2} \bar{T}=\frac{1}{2\left(c+c^{\prime}\right) \rho_{0}} \int_{-z_{0}}^{z_{0}}\left[a\left(1-b c^{\prime} P_{0}\right)+\frac{c^{\prime}}{2\left(c+c^{\prime}\right) \rho_{0}} z^{2}\right] \sqrt{z_{0}^{2}-z^{2}} d z
$$

which gives

$$
\rho_{0} \bar{T}=a\left(1-b c^{\prime} P_{0}\right) \frac{\left(z_{0} / \rho_{0}\right)^{2}}{4\left(c+c^{\prime}\right)}+\frac{c^{\prime} \rho_{0}}{2}\left[\frac{\left(z_{0} / \rho_{0}\right)^{2}}{4\left(c+c^{\prime}\right)}\right]^{2}
$$


Expressing $z_{0}$ in terms of $P_{0}$ by (10), this equation gives the following to determine the one unknown $\left(\frac{a P_{0}}{\rho_{0} \bar{T}}\right)$

$$
\frac{a P_{0}}{\rho_{0} \bar{T}}=1+\frac{\rho_{0} c^{\prime} \bar{T}}{a}\left(b-\frac{\rho_{0}}{2 a}\right)\left(\frac{a P_{0}}{\rho_{0} \bar{T}}\right)^{2}
$$

According to the assumptions underlying this derivation $\frac{\rho_{0}}{a}$ is of the order of magnitude of, say, $10^{-3}$, while $c$ and $c^{\prime}$ are about $10^{-12}$, while the average tension of the wire, $\bar{T}$, is say $(10)^{9}$ dyne $\mathrm{cm}^{-2}$ (corresponding to a total tension of 7 pounds). Consequently the term $\frac{\rho_{0} c^{\prime} T}{a}$ is $10^{-6}$, which shows (since $b$ is of the order of magnitude of unity) that $\frac{a P_{0}}{\rho_{0} \bar{T}}=1$ (to 1 part in $1,000,000$ ). We have, therefore, the final value of $P_{0}$.

$$
P_{0}=\frac{\rho_{0} \bar{T}}{a^{-}}
$$

whence the final value of the width $2 z_{0}$ of the contact ring becomes

$$
2 z_{0}=4 \rho_{0} \sqrt{\left(c+c^{\prime}\right) \frac{\rho_{0} \bar{T}}{a}}
$$

The equation of the contact ring becomes

$$
r(z)=a-\rho_{0} c^{\prime} \bar{T}\left[b-2 \frac{\rho_{0}}{a}\left(\frac{z}{z_{0}}\right)^{2}\right]
$$

where the $b$ is to be computed by the formula

$$
b=\frac{\rho_{0}}{2 p\left(1+\sigma^{\prime}\right)}+\frac{\rho_{0}}{a}\left[1+\log \frac{a \rho^{2}}{\rho_{0}{ }^{3}\left(c+c^{\prime}\right) \bar{T}}\right]
$$

The pressure distribution is given by equation (8). The remaining constant $a_{0}$ (the initial distance of wire center from $z$ axis) is found by eliminating $A$ between (6) and (9) to be [neglecting 1 part in 1,000,000; that is, $(c \bar{T})^{2}$ compared to 1]

$$
a_{0}=a+\rho_{0}-a \lambda_{1} c \bar{T}^{\cdots} \rho_{0}\left[b c^{\prime} \bar{T}+\left(\lambda_{1}+\lambda_{2}\right) c \bar{T}\right]
$$

The shape of the cylinder at points outside a contact ring such as for $z_{0}<z<2 \pi p-z_{0}$ may now be found by using the values of $P_{0}$ and $P(z)$ from (15) and (8) in the equation (79) of Section IV. This leads to

$$
\begin{gathered}
r(z)=a-\rho_{0} c^{\prime} \bar{T}\left\{\frac{\rho_{0}}{2 p\left(1+\sigma^{\prime}\right)}+\frac{2 \rho_{0}}{a}[\log p\right. \\
\left.\left.-\frac{2}{\pi z_{0}} \int_{-z_{0}}^{z_{0}} \sqrt{1-\left(\frac{z^{\prime}}{z_{0}}\right)^{2}} \log \left(2 \rho \sin \frac{z-z^{\prime}}{2 \rho}\right)\right]\right\}
\end{gathered}
$$


Now

$$
\begin{gathered}
\int_{-z_{0}}^{z_{0}} \sqrt{1-\left(\frac{z^{\prime}}{z_{0}}\right)^{2}} \log \left(2 p \sin \frac{z-z^{\prime}}{2 p}\right)=\frac{\pi z_{0}}{2} \log 2 p \\
+\int_{-z_{0}}^{z_{0}} \sqrt{1-\left(\frac{z^{\prime}}{z_{0}}\right)^{2}} \log \left(\sin \frac{z}{2 p} \cos \frac{z^{\prime}}{2 p}-\cos \frac{z}{2 p} \sin \frac{z^{\prime}}{2 p}\right) d z^{\prime}
\end{gathered}
$$

When the point $z$ is near the halfway point between the two wires, $\sin \frac{z}{2 p}$ is near unity and $\cos \frac{z}{2 p}$ is small, while $\cos \frac{z^{\prime}}{2 p}$ is near unity and $\sin \frac{z^{\prime}}{2 p}$ is small. Hence (as before), by neglecting second-order terms of the order of $\left(\frac{z_{0}}{p}\right)^{2}$ we find

$$
\int_{-z_{0}}^{z_{0}} \sqrt{1-\left(\frac{z^{\prime}}{z_{0}}\right)^{2}} \log \sin \frac{z-z^{\prime}}{2 p} d z^{\prime}=\frac{\pi z_{0}}{2} \log \sin \frac{z}{2 p}
$$

which is a good approximation if $z$ is not very close to a contact surface. Hence, the shape of the cylinder at points not very close to a contact ring is found by equation (20) to be very approximately

$$
r(z)=a-\rho_{0} c^{\prime} \bar{T}\left[\frac{\rho_{0}}{2 p\left(1+\sigma^{\prime}\right)}-\frac{2 \rho_{0}}{a} \log 2 \sin \frac{z}{2 p}\right]
$$

This shows that at a point midway between two wires, where, say, $z=\pi p$

$$
r(\pi p)=a-\rho_{0} c^{\prime} \bar{T}\left[\frac{\rho_{0}}{2 p\left(1+\sigma^{\prime}\right)}-\frac{\rho_{0}}{a} \log 4\right]
$$

This is the thickest part of the cylinder. Its thinnest part is at $z=0$, where $r=r(0)$. The difference between its greatest and least radius is found by (17) and (22) to be

$$
r(\pi p)-r(0)=\rho_{0} c^{\prime} \bar{T} \frac{\rho_{0}}{a}\left[1+\log \frac{4 a p^{2}}{\rho_{0}{ }^{3}\left(c+c^{\prime}\right) \bar{T}}\right]
$$

This is seen to be small compared to the compression at the mid-point which by (22) may be written

$$
a-r(\pi p)=\rho_{0} c^{\prime} \bar{T}\left[\frac{\rho_{0}}{2 p\left(1+\sigma^{\prime}\right)}-\frac{\rho_{0}}{a} \log 4\right.
$$

Hence, the cylinder is almost uniformly compressed with relatively shallow depressions where the wires touch it.

To find the deformations of the wires, we must use the values here found $P_{0}=P_{1}=\frac{\rho \bar{T}}{a}$ in the equation (42) of Section III, which gives

$$
\frac{C}{4 \epsilon}=\bar{T}\left\{1+\frac{\rho_{0}}{a}\left[\sigma-\frac{\rho_{0}}{4 a}\left(\frac{1+2 \sigma-7 \sigma^{2}}{1-\sigma}\right)\right]\right\}
$$


If we neglect terms of the order of 1 part in $1,000,000$ and consider $\frac{\rho_{0}}{a}$ and $c T$, as each of the order of $10^{-3}$, then this becomes

$$
\frac{C}{4 \epsilon}=\bar{T}\left\{1+\frac{\rho_{0} \sigma}{a}\right\}
$$

The components of the displacement in the wire corresponding to the stress function $\chi$ are, therefore, of the order of $10^{-12}$ and may be neglected, but the particular solution of Section III gives by equation (24)

$$
\begin{gathered}
u_{\rho}\left(\rho_{0}, \beta\right)=-\frac{\sigma a_{0} \bar{T} \rho_{0}}{E a}-a_{0} c \bar{T} \lambda_{1}\left(1+\frac{\rho_{0} \sigma}{a}\right) \cos \beta \\
u_{\beta}\left(\rho_{0}, \beta\right)=a_{0} c \bar{T} \lambda_{1}\left(1+\frac{\rho_{0} \sigma}{a}\right) \sin \beta
\end{gathered}
$$

The cylindrical components of this displacement of points initially on a circular section of the wire are

$$
\begin{gathered}
u_{r}\left(\rho_{0}, \beta\right)=a_{0} c \bar{T} \lambda_{1}\left(1+\frac{\rho_{0} \sigma}{a}\right)+\frac{\sigma a_{0} \bar{T} \rho_{0}}{E a} \cos \beta \\
u_{z}\left(\rho_{0}, \beta\right)=\frac{\sigma a_{0} \bar{T} \rho_{0}}{E a} \sin \beta
\end{gathered}
$$

In these four equations $a_{0}$ may be replaced in terms of given constants by means of (19).

The two equations (26) and (27) show that the points on the circular section of the wire suffer a uniform contraction of radius, together with a translation in the $r$ direction, both of which leave its section circular. The amount of the contraction of radius of the wire is given by

$$
\rho_{0}-\rho=\frac{\sigma \bar{T} a_{0} \rho_{0}}{E a}=\frac{\sigma \bar{T} \rho_{0}}{E}
$$

The translation is much larger, being

$$
u_{r}\left(\rho_{0}, \frac{\pi}{2}\right)=a_{0} c \bar{T} \lambda_{1}\left(1+\frac{\rho_{0} \sigma}{a}\right)
$$

The result reached in equation (30) is of interest in absolute measurements in which the inductance of a single-layer solenoid is computed from measurements of the diameter of the solenoid (over the windings), its length and the pitch of the windings, and from the radius $\rho_{0}$ of the wire measured before it was wound. The formula for the inductance requires a knowledge of the radius $a^{\prime}$ of a current sheet which passes through the centers of the wires. If $D$ is the measured over-all diameter, this radius is $a^{\prime}=\frac{D}{2}-\rho=\frac{D}{2}-\rho_{0}+\sigma \frac{\bar{T}}{E} \rho_{0}$. For the solenoid $\frac{D}{2}=14.5 \mathrm{~cm}$ approximately; $\rho_{0}=0.03545 \mathrm{~cm} ; \sigma=\frac{1}{3}$; 
$E=1.2(10)^{12}$ and $\bar{T}=8(10)^{8}$ dyne $\mathrm{cm}^{-2}$, since the wire was wound with a tension of 7 pounds. Hence, $\sigma \bar{T} \bar{T} \rho_{0}=8(10)^{-6} \mathrm{~cm}$, so that the correction due to contraction of the wire alters the radius of the current sheet by 1 part in $2,000,000$.

\section{SUMMARY}

It has been found that in the case of a long circular cylinder of radius $a$, uniformly wound with equal, and equally spaced, wires of radius $\rho_{0}$, each with tension $T$, that the pressure distribution under any wire (such as the one whose center is in the plane $z=0$, the $z$ axis being the axis of the cylinder) is

$$
P(z)=\frac{\sqrt{z_{0}^{2}-z^{2}}}{2\left(c+c^{\prime}\right) \rho_{0}} \text { for }-z_{0}<z<z_{0}
$$

where the width of the contact ring $2 z_{0}$ is given by

$$
2 z_{0}=4 \rho_{0} \sqrt{\left(c+c^{\prime}\right) \frac{\rho_{0} \bar{T}}{a}}
$$

where $\bar{T}=T / \pi \rho_{0}{ }^{2}=$ average tension in the wire, and

$$
c=\frac{1-\sigma^{2}}{E}, \quad c^{\prime}=\frac{1-\sigma^{\prime 2}}{E^{\prime}}
$$

$\sigma$ and $E$ being Poisson's ratio and Young's modulus, respectively, for the wire material, while $\sigma^{\prime}, E^{\prime}$ refer to the material of the cylinder. If the constant $b$ is defined by equation (18) of Section V, the shape of the dent in the cylinder in which a wire makes contact is

$$
r(z)=a-\rho_{0} c^{\prime} \bar{T}\left[b-\frac{2 \rho_{0}}{a}\left(\frac{z}{z_{0}}\right)^{2}\right] \text { for }-z_{0}<z<z_{0}
$$

where $r(z)$ denotes the distance from the $z$ axis to the surface of the cylinder. At points on the surface of the cylinder outside a contact ring, and distant from the edges of one by a quantity which is not small of the order of $z_{0}$, the shape of the cylinder is given by the periodic function of $z$

$$
r(z)=a-\rho_{0} c^{\prime} \bar{T}\left[\frac{\rho_{0}}{2 p\left(1+\sigma^{\prime}\right)}-\frac{2 \rho_{0}}{a} \log \left(2 \sin \frac{z}{2 p}\right)\right]
$$

where $2 \pi p$ is the distance between centers of adjacent turns of wire. The cylinder is almost uniformly compressed, with a relatively small bulge between the wires. The circular section of the wire undergoes a practically uniform contraction of radius, $\rho_{0}-\rho=\frac{\sigma \bar{T}}{E} \rho_{0}$, together with a translation in the $r$ direction (away from the axis of the cylinder) of amount $a_{0} c \bar{T} \lambda_{1}\left(1+\frac{\rho_{0} \sigma}{a}\right)$ where $\lambda_{1}=\frac{1-2 \sigma-\sigma^{2}}{(1+\sigma)(1-\sigma)^{2}}$ and where $a_{0}$ is the distance of the center of the wire section from the axis of the cylinder when the turn of wire is unstressed. It is given by equation (19) of Section V. 
It is believed that this evaluation of the pressure distribution is new. It differs from that of H. Hertz ${ }^{4}$ in two respects. In Hertz's problem the area of contact was the infinitesimal neighborhood of a point so that its boundary was found to be an infinitesimal ellipse, and the integral equation determining the pressure distribution a Newtonian potential of that distribution. In the present problem this area has one large dimension, since it is a ring of small width $2 z_{0}$ upon the face of the cylinder and the integral equation determining the pressure distribution is its logarithmic potential, a difference which seems natural.

The other point of difference is that Hertz considered the pressure to be so local in its effect that the stress and strain produced by it were confined to its immediate neighborhood so that he considered the two bodies as separated by an infinite plane. This magnification of the region so simplified his problem that the pressure and the pressure ellipse could be evaluated without reference to the actual shapes of the two bodies. However, as all points in this infinite plane were in reality points very close to the contact ellipse, his results (stress and strain) were only applicable to pointsin the immediate neighborhood of the contact. The problem here solved is considerably more general for we first formulated the elastic displacement at all points in a wire in terms of an integral over the pressure area, involving the unknown pressure distribution, which satisfied the condition of no stress at all other parts of the boundary of the wire. Only when the point was on the contact area did this integral reduce to a (logarithmic) potential integral. Similarly the elastic displacement at any point in the cylinder was formulated in terms of an integral involving the pressures of all the wires, which displacement satisfied the condition of no stress at points on the cylindrical surface between contact areas. When the point on the cylinder approached a point on a contact surface, a potential integral again appeared. By expressing the fact of contact between wires and cylinder, an integral equation resulted which determined the pressure distribution and its range. This being found, we have determined the deformation and stress not only in the immediate neighborhood of the contact area, but at all points in wires and cylinder. It must be pointed out, however, that the results here obtained are by no means exact solutions of the elastic equations. They are approximations limited by the fact that the ratio $\frac{\rho_{0}}{a}$ of the radii of wire and cylinder are small, and three relative orders of small quantities $\frac{a}{\rho_{0}}$, $1, \frac{\rho_{0}}{a}$ are retained. The problem has also been limited to the case where the pitch of the windings $2 \pi p$, (that is, distance between wires) is of the same order as the radius of a wire, but the removal of this restriction is obviously very easy, as it would not involve any modification of the solution for points in a wire, but only for points in the cylinder, and for this part of the problem we obtained an exact solution.

Washington, March, 1930.

4 H. Hertz, J. f. Math. (Crelle), Bd. 92; 1881. 